\title{
Pour un développement rurbain durable
}

\author{
Antoine Bailly \\ Université de Genève
}

\section{Une vague de fond : le rurbain ${ }^{1}$}

Qui aurait pu penser, au début du siècle, à l'ampleur du développement de l'habitat et des activités dans les zones anciennement rurales, devenues «rurbaines» selon le terme reconnu dans les statistiques? Ni la théorie des lieux centraux de Christaller ni la théorie des pôles de croissance de Perroux n'envisageaient ce dynamisme propre au rurbain, tant l'accent était mis sur le seul rôle des villes dans la croissance.

Seuls certains architectes comme Wright ${ }^{2}$, avec son projet de broadacres présenté au MoMA (Museum of Modern Art) de New York dans les années 1930, une dissémination organisée du pavillonnaire sur le territoire des États-Unis, avec un maillage routier adapté, et les «désurbanistes 》 soviétiques des années 1920 proposant un urbanisme de faible densité (en opposition avec les urbanistes et leur habitat collectif) envisageaient cette déconcentration en la structurant. Mais le germe existait un peu partout dans le monde avec le mythe jeffersonien de la vie en communauté, ou dans les projets d'unités de voisinage qui proposaient de regrouper les habitants en unités de l'ordre de 2000 habitants avec leurs services de voisinage, des sortes de villages, ce qui fut fait dans de nombreuses villes nouvelles, en Angleterre par exemple, et dans les banlieues américaines.

$\mathrm{Au}$ début du troisième millénaire, le mythe de la vie villageoise se renforce dans les sociétés occidentales et la rurbanisation s'accélère, utilisant les aires accessibles et attrayantes par leur environnement social et naturel et par leur image positive bien au delà du suburbain. La mobilité accrue et le télétravail renforcent le mouvement. Ce processus modifie profondément la vie sociale, économique et les pouvoirs politiques dans le rurbain.
On oublie souvent que le «non urbain », comme on le dit encore dans certains ouvrages, couvre $60 \%$ de l'espace en France et le « péribain », 33 \%. La proportion est similaire dans les pays d'Europe occidentale. Il y a donc de l'espace à aménager à une époque où le monde rural, après une période de crise, attire résidents et activités et évolue de la monoactivité vers le multifonctionnel. Mais il faut dire qu'à part la politique de valorisation des villes moyennes dans les années 1980, ou les schémas d'aménagement de régions périphériques comme la Gaspésie au Québec dans les années 1960, priorité a été donnée aux politiques urbaines (métropoles d'équilibre) et aux grandes métropoles. Même les politiques agricoles de l'Union européenne restent axées sur l'aide aux productions, sans effet sur l'aménagement du rurbain.

Le rurbain a en effet mauvaise presse! En négatif, on assiste au déclin de l'agriculture traditionnelle ou à la fin des industries rurales et au mitage du paysage par un pavillonnaire désorganisé. En positif, pourtant, il y a attraction de nouvelles populations dans des environnements agréables tant sur le plan paysager que social... Les promoteurs ne s'y trompent pas et lancent leurs programmes à succès autour des villages ou même dans des zones libres de constructions. Une nouvelle société rurbaine naît, avec ses nouvelles formes d'habiter, ses nouvelles activités de services, ses nouvelles pratiques sociales et de mobilité. Que faire, donc, pour mieux encadrer ce mouvement inéluctable?

\section{Valoriser les ressources du rurbain}

Le rurbain, comme tout territoire, constitue un espace enjeu entre de multiples acteurs mobilisant activités et ressources. La théorie des milieux ${ }^{3}$ permet de comprendre les logiques de construction de représenta- 
tions de règles communes et de projets collectifs dans ces territoires.

Le rôle des différents acteurs locaux est essentiel pour une valorisation du rurbain. En effet, si, il y a 50 ans, le monde rural était surtout celui des agriculteurs, ce même milieu est maintenant investi par de multiples usagers qui l'occupent et parfois le contrôlent. Gelin ${ }^{4}$ propose une typologie des ces usagers selon :

- l'ancienneté de l'ancrage dans le milieu, qui différencie populations agricoles des nouveaux résidents;

- les modalités d'usage du milieu, qui dissocient les producteurs, les résidents principaux, les résidents secondaires;

- les rapports à la ville d'anciens citadins retraités, des actifs urbains et des actifs rurbains;

- l'implication plus ou moins forte dans les politiques locales.

Chaque acteur se positionne dans ces catégories, ce qui introduit une grande complexité dans ce paysage social puisque les producteurs agricoles impliqués voient leur position diminuer, alors que les résidents actifs et retraités s'accroissent. Et Gelin parle de profils principaux comme les nostalgiques du rural ancien, les nouveaux enracinés qui s'impliquent localement ou les « sans territoire » qui viennent avant tout chercher un environnement calme et sécuritaire.

Torre ${ }^{5}$, dans une série d'études sur les tensions et conflits dans le rurbain, montre comment ceux-ci sont sources de changement dans la gestion de ce milieu. Les structures de gouvernance - hiérarchie des acteurs, règles de fonctionnement - se modifient lentement, et les dispositifs locaux évoluent, parfois dans la crise, malgré les inerties. Les majorités changent, intégrant de nouveaux rurbains, parfois étrangers, et le conflit devient créateur de nouveaux pouvoirs et de liens sociaux.

Dans ces territoires, comme le montre le programme de l'Institut national de la recherche agronomique « Pour et sur le développement régional ${ }^{6}$, la multifonctionnalité devient la règle avec trois usages majeurs $^{7}$ :
- l'usage productif par mobilisation des ressources locales (agriculture, forêt, énergie, matières premières, espace...);

- l'usage de nature par préservation de l'eau, des sols, de la biodiversité et la prévention des risques;

- l'usage résidentiel, de loisirs et d'autres activités (services, artisanat, industrie).

Ce troisième usage prend de l'importance, ce qui, par conséquent, rend le deuxième usage plus nécessaire. De véritables noyaux d'activités de services et de loisirs se créent. Dans ces territoires, le mélange d'acteurs rend nécessaires de nouvelles politiques locales. Dans un contexte de réforme des fonds structurels européens à l'horizon 2007, qui s'accompagne d'un renforcement de la régionalisation (logique de subsidiarité), le rurbain va continuer à se transformer et à prendre de l'ampleur. Il faut donc prévoir l'encadrement nécessaire à la maîtrise de l'étalement spatial des activités et la préservation de l'environnement.

\section{Une composante majeure du rurbain est son accessibilité.}

\section{Vers une nouvelle organisation du rurbain}

Un changement profond dans la conception du rurbain est devenu indispensable. Le « tout urbain », avec ses politiques restrictives tant sur le plan urbanistique que sur celui des transports individuels, pousse de nombreuses familles dans le rurbain. L'insécurité et le chômage urbain renforcent cette tendance. Mais le rurbain a besoin de politiques claires sur le plan des transports, des services et de la gouvernance des territoires s'il veut conserver son attractivité.

\section{Les transports et l'accessibilité}

Une composante majeure du rurbain est son accessibilité. Une région périphérique mal reliée aux centres majeurs est handicapée dans son développement. Mais il faut également penser à l'accessibilité intrarurbaine puisque des emplois, des activités de services et de loisirs s'y implantent.

À la vision fonctionnaliste des transports vers les centres pour le travail se substitue une conception plus 
large des déplacements individuels et collectifs. La notion de congruence est fondamentale dans ce domaine, car les effets structurants des infrastructures de transport se produisent dans un système territorial qu'elles vont modifier, influençant le développement de certains secteurs et en retardant d'autres.

Une gestion durable des transports dans le rurbain est celle qui prévoit la bonne mobilité des personnes et des informations à l'accessibilité intra-rurbaine, y compris en transports individuels et aux répercussions de la création d'infrastructures sur la qualité de vie de la population et son environnement. Or, actuellement, les différents systèmes de transport sont gérés par des acteurs multiples privilégiant leurs logiques propres. En résulte une complexité et un enchevêtrement de fonctionnement nuisible à un développement du rurbain équilibré. Une conception globale des transports et de l'accessibilité est devenue aussi indispensable dans le rurbain que dans l'urbain. Les désurbanistes et les promoteurs du développement dans ce milieu y ont réfléchi; les autorités politiques se contentent de suivre lorsque les routes sont saturées ou que les transports en commun sont trop dégradés ou inexistants...

\section{Pour le secteur public, le défi est difficile : fournir des services de qualité, selon des critères précis, avec des ressources en baisse.}

\section{Valoriser les services publics}

Le bon fonctionnement des services publics constitue une autre composante majeure de la qualité de vie du rurbain. Non seulement ils contribuent à l'attrait des territoires, mais ils génèrent des effets économiques : de bons services comme de bonnes infrastructures favorisent la vitalité économique et sociale. De nombreuses enquêtes le montrent, en particulier pour les services postaux, bancaires, hospitaliers et les transports.

Pour le secteur public, le défi est difficile : fournir des services de qualité, selon des critères précis, avec des ressources en baisse. Or, si ces services n'atteignent pas les niveaux de qualité, la légitimité même des collectivités publiques est remise en question. «Faire plus avec moins » ou « faire autrement», tels sont les éléments de ce défi pour le rurbain. De ce point de vue, il faudrait que les services publics se comportent comme des entreprises privées; mais leur mission est très souvent non rentable. Il faut donc dissocier les services susceptibles d'être lucratifs de ceux qui ne peuvent l'être.

Pour les entreprises publiques qui fonctionnent de manière compétitive, une réorganisation est nécessaire pour intégrer les demandes du rurbain; pour celles qui ne le peuvent pas, il devient indispensable de réfléchir à leur culture organisationnelle et à leur légitimité. Tel est le cas, par exemple, des services sociaux ou de transport en commun en zones de faible densité.

Pour définir des services publics de qualité, il faut préciser leurs domaines d'intervention, les résultats attendus ainsi que les ressources financières à mobiliser. Des contrats peuvent être passés avec les services concernés pour évaluer les niveaux de réalisation des objectifs. Il s'agit de valoriser la notion de service public et de dynamiser son image : efficacité, flexibilité, cohésion de l'organisation, capacité de créativité constituent des éléments de cette valorisation, dans un contexte d'équité sociale et spatiale et de respect des intérêts de la société civile.

\section{Un habitat dans un contexte durable}

Dans la gestion de l'exurbanisation et de la rurbanisation, les politiques foncières et d'habitat constituent, de longue date, un autre élément de l'intervention publique. C'est également un domaine majeur des interventions publiques.

À une période d'arrivée de populations nouvelles dans le rurbain, ces politiques prennent une ampleur particulière pour éviter le développement anarchique et le mitage résidentiel. Elles s'inscrivent nécessairement dans un contexte de multifonctionnalité : le système habitat est très dépendant des autres systèmes en interaction : économiques, sociaux, culturels...

Le rôle des acteurs publics est restreint à court terme; mais à plus longue échéance, il est possible d'envisager des effets durables de politiques intégrant la dimension du temps long. Planifier l'habitat et les infrastructures signifie évaluer le système actuel, envisager son évolution en fonction des interactions avec d'autres systèmes et penser aux futurs possibles dans un contexte durable, respectueux de l'environnement. 


\section{Une nouvelle gouvernance locale}

Comment gérer des territoires découpés selon de multiples structures institutionnelles et spatiales héritées du passé, avec leurs compétences et leurs intérêts divergents? Les formes de la gouvernance locale sont au centre de débats multiples sur la nécessité de politiques territoriales cohérentes, respectueuses de la diversité des communautés. Y a-t-il des contradictions insolubles dans les conflits que nous avons évoqués?

L'émergence de mouvements sociaux et de groupes communautaires constitue le signe d'un renouveau dans la gestion actuelle du rurbain. Ils posent la question de l'efficacité des gouvernements locaux et de leur potentiel à résoudre des problèmes généraux ou locaux, tels ceux liés à la multifonctionnalité.

La façon de gérer le rurbain évolue donc rapidement $\mathrm{du}$ fait des contradictions dans le système. Une nouvelle gouvernance globale est devenue indispensable pour mieux prévoir le futur du rurbain. Cette gouvernance suppose des approches transversales entre les différentes politiques, des réflexions à plusieurs échelles géographiques et temporelles et la formalisation de règles de mise en œuvre des politiques entre partenaires. Il est alors indispensable de réfléchir en termes d'acteurs, d'échelles et de contextes pour promouvoir dans chaque cas l'équité, l'efficacité et la durabilité. C'est ainsi que peuvent être dégagées des priorités de planification articulant et associant des institutions politiques, des acteurs sociaux et des organisations publiques et privées dans des processus d'élaboration et de mise en œuvre de choix collectifs capables de provoquer une adhésion-action des citoyens.

\section{Une coopération sociale et culturelle}

Un volet particulièrement important dans les politiques $\mathrm{du}$ rurbain concerne leurs aspects sociaux et culturels, souvent mis au deuxième plan derrière des logiques économiques et budgétaires. Pourtant, le capital social existe avec ses modes de fonctionnement, ses règles, ses réseaux indispensables à la qualité de vie; il permet la valorisation des spécificités locales et du sens communautaire. Les institutions sociales servent ainsi à l'intégration de ceux qui pourraient être exclus des sociétés rurbaines modernes et à la valorisation de chaque usager (ce qui n'est plus le cas dans les milieux urbains).
Dans des sociétés où le rôle de la famille s'estompe, les politiques sociales et culturelles jouent un rôle majeur pour la valorisation des identités locales. Valorisation du local pour faire vivre les territoires et pour créer une qualité de vie et une image positive dans un ensemble cohérent. Il s'agit, dans chaque cas, de prendre en compte la dimension territoriale des politiques, d'intégrer l'ensemble des acteurs à des échelles géographiques différentes et de prévoir les politiques sociales et culturelles en termes d'équité et de durabilité. $\mathrm{Au}$ lieu d'une gestion verticale descendante, ces politiques peuvent être assises sur la vitalité du local et associer la société civile à l'action publique.

Les institutions sociales servent ainsi à l'intégration de ceux qui pourraient être exclus des sociétés rurbaines modernes.

\section{Pour conclure : le rurbain, un espace à qualité de vie pour l'avenir}

Voir le rurbain comme un milieu économique et social, producteur de richesses, telle est la vision que nous souhaitons développer. Au-delà des pures logiques fonctionnelles, cette vision part de la volonté de développement durable de ces milieux. Acteurs et territoires sont liés lors de la mise en place de projets de développement et d'aménagement. Les chances de conserver et d'attirer de façon durable des hommes et des activités sont liées à ce contexte dynamique et coopératif. L'ensemble des autres politiques, décrites précédemment, contribue également à ce dynamisme, car il n'y a pas de développement sans services publics ou transport de qualité...

Les propositions de ce texte ne constituent pas des recettes mais des pistes apparues à la suite des débats scientifiques récents, en particulier à la DATAR (Délégation à l'aménagement du territoire et à l'action régionale) et à l'INRA (Institut national de la recherche agronomique) en France. Elles ont pour objectif de clarifier les besoins actuels et futurs du rurbain. Pour envisager la mise en place de politiques concrètes dans un contexte de développement durable, elles visent aussi à dépasser les pratiques non adaptées à ce contexte.

Les pouvoirs sont plus que jamais mis au défi d'agir efficacement dans une conjoncture incertaine. Nul ne 
peut plus décider sans s'être assuré des conséquences futures des choix et sans y associer l'ensemble des acteurs. La responsabilité et la légitimité des pouvoirs territoriaux rurbains dépendent de leur potentiel à maîtriser l'avenir par un aménagement du territoire de qualité et durable. Grâce à une nouvelle gouvernance, intégrant ainsi développement économique, social, culturel et environnemental, l'avenir peut, plus que jamais, être envisagé de façon positive.

\section{Notes et références}

1 Dans cet article, nous distinguons l'exurbain, prolongation diffuse du suburbain, du rurbain intégré dans le milieu rural souvent dans des villages anciens, parfois nouveaux ou en habitat pavillonnaire disséminé!

2 Wright F.L. (1958). The living city, New York, Horizon Press.
3 Camagni R. et D. Maillat (dir.) (2006). Milieux innovateurs, Paris, Economica.

4 Gelin S. (2006). « De la parcelle aux territoires... », dans Territoires, Acteurs et Agriculture en Rhône-Alpes, Lyon, INRA. http://www.inst-elevage.asso.fr/html31/IMG/pdf/ Synthese_Axe_2_DEF.pdf

5 Torre A. (2006). « Tensions et conflits des différents usages des espaces ruraux », dans Territoires, Acteurs et Agriculture en Rhône-Alpes, Lyon, INRA.

6 INRA, 2007, Programme « Pour et sur le développement régional » 2007-2010, Grenoble, INRA.

http://www.inra.fr/les_partenariats/collaborations_et_parte naires/monde_agricole_et_rural/typologie_des_actions/pro grammes_pour_et_sur_le_developpement_regional/psdr_3

7 Perrier-Cornet P. (2001). « La dynamique des espaces ruraux dans la société française : un cadre d'analyse », Territoires 2001, $\mathrm{n}^{\circ} 3$, p. 64-71. 


\section{Publicité}

CRDT 